\title{
Sistema de pulsioximetría y capnografía para dispositivos móviles Android
}

\author{
Carlos Andrés Gómez García ${ }^{*}$, Jaime Velasco Medina \\ Grupo de investigación Bionanoelectrónica, Escuela de Ingeniería Eléctrica y Electrónica, \\ Universidad del Valle, Colombia
}

Recibido 4 de septiembre de 2013. Aprobado 12 de febrero de 2014

\section{A PULSE-OXIMETRIC AND CAPNOGRAPHIC SYSTEM FOR ANDROID MOBILE DEVICES}

\author{
SISTEMA DE OXIMETRIA DE PULSO E CAPNOGRAFIA PARA DISPOSITIVOS MÓVEIS ANDROID
}

\begin{abstract}
Resumen - El monitoreo constante del nivel de saturación de oxígeno y la producción de $\mathrm{CO}_{2}$ es de vital importancia para la supervisión del estado respiratorio del paciente. Este artículo presenta el diseño de un sistema de oximetría de pulso y capnografía que tiene como unidad de procesamiento un chip programable de señales mixtas denominado PSoC (Programable-System-OnChip), el cual incorpora bloques análogos y digitales configurables, permitiendo que la adecuación de las señales suministradas por los sensores y el procesamiento digital de señales se lleve a cabo en el mismo chip. Se realizó una aplicación en Android para la visualización y registro de las señales biomédicas en una base de datos local, compatible con dispositivos móviles con conectividad wifi. El sistema fue verificado usando un simulador de $\mathrm{SpO}_{2}$ (Saturación parcial de oxígeno), que permitió la calibración de frecuencias cardiacas desde 55 BPM (Beats per Minute) a $145 \mathrm{BPM}$, así como la curva R con valores de $75 \%$ a $100 \%$ de $\mathrm{SpO}$. Se encontró que el error de medición de la frecuencia cardiaca es 1,81\%, y $1.33 \%$ para la $\mathrm{SPO}_{2}$.
\end{abstract}

Palabras Clave - Android; Capnografía; PSoC; Pulsioximetría; Señales biomédicas; Sistemas embebidos.

Abstract - Constant monitoring of oxygen saturation level and $\mathrm{CO}_{2}$ production is vital for monitoring the patient's respiratory status. This paper presents the design of a pulse-oximetric and capnographic system, which core consists of a mixed signal programmable chip, PSoC (Programmable-System-On-Chip), which incorporates a whole analog and digital configurable block system, in order to adequate and process the signals from the sensors all in a single chip. An Android application was also developed, which can display biomedical signals in mobile devices with wireless connectivity, as well as to store information from these signals in a local user database. The microsystem was verified using a $\mathrm{SpO}_{2}$ (oxygen partial saturation) simulator, and heart rates of $55 \mathrm{BPM}$ to $145 \mathrm{BPM}$ were calibrated, as well as the R curve with values of $75 \%$ to $100 \% \mathrm{SpO}_{2}$. The heart rate measurement error found is $1,81 \%$ and $1,33 \%$ for the $\mathrm{SpO}_{2}$.

Keywords - Android; Biomedical signals; Capnography; Embedded systems; PSoC, pulse oximetry.

\footnotetext{
\% Dirección para correspondencia: carlosgo515@gmail.com DOI: http:/dx.doi.org/10.14508/rbme.2014.8.15.36-44
} 
Resumo - O monitoramento constante do nível de saturação de oxigênio e produção de $\mathrm{CO}_{2}$ é fundamental para monitorar o estado respiratório do paciente. Este artigo apresenta o projeto de um sistema de oximetria de pulso e capnografia cuja unidade de processamento um chips de sinal misto programável chamado PSoC (Programmable-System-On-Chip), o qual incorpora blocos analógicos e digitais configuráveis, permitindo a adaptação dos sinais fornecidos pelos sensores e o processamento digital de sinais será executada no mesmo chip. Foi realizada una aplicação Android para visualização e gravação de sinais biomédicos em um banco de dados local, compatível com dispositivos móveis com conectividade sem fio. O sistema foi testado usando um simulador de $\mathrm{SpO}_{2}$ (saturação de oxigênio parcial), permitindo a calibração da freqüência cardíaca de 55 BPM (batidas por minuto) a 145 BPM, assim como a curva $\mathrm{R}$ com valores de $75 \%$ a $100 \% \mathrm{SpO}_{2}$. Verificou-se que o erro de medição do ritmo cardíaco é $1,81 \%$ e $1,33 \%$ para o $\mathrm{SPO}_{2}$.

Palavras-chave - Android; capnografia; PSoC; oximetria de pulso; Sinais biomédicos; Sistemas Embebidos.

\section{INTRODUCCIÓN}

T as señales biomédicas contienen información que puede ser utilizada para entender algunos mecanismos fisiológicos subyacentes en un sistema biológico específico [1]. El monitoreo ambulatorio de señales biomédicas tales como la saturación de oxígeno en la sangre $\left(\mathrm{SpO}_{2}\right)$, las pulsaciones por minuto, la presión parcial de $\mathrm{CO}_{2}$ final expirada $\left(\mathrm{EtCO}_{2}\right)$ y la frecuencia respiratoria, es un importante procedimiento de supervisión, que permite proporcionar información valiosa sobre el estado de los pacientes en riesgo de hipoxia o ventilación inadecuada [2]. Una supervisión continua de estas señales y parámetros biomédicos es de vital importancia para conocer el estado respiratorio del paciente, muy útil en la detección de complicaciones respiratorias como la AOS (Apnea Obstructiva del Sueño), en la que la respiración se reduce o se detiene brevemente mientras el paciente duerme.

Los equipos presentes en el mercado colombiano que permiten la visualización, monitorización y registro de múltiples signos vitales son muy especializados y bastante costosos. Sin embargo, el desarrollo de sistemas mHealth (mobile health) es una gran opción para usar este tipo de equipos de vital importancia para el cuidado de la salud.

Un estudio publicado en la revista Anesthesia \& Analgesia [3] sugiere que el uso de estos equipos como dispositivos médicos podría revolucionar el cuidado de nuestra salud. En particular, los investigadores discutieron la posibilidad de que smartphones y tablets pudieran ser utilizados en la pulsioximetría, la cual no es muy utilizada en los países en vías de desarrollo, debido a que la implantación de la pulsioximetría tiene importantes costes económicos. Sin embargo, el uso de mHealth para desarrollar este procedimiento no invasivo en los hospitales y centros médicos de estas regiones podría facilitar su uso.

Investigadores de la Universidad de Chicago [4] aseguran que los médicos serían más eficientes en su práctica clínica si utilizaran tablets o smartphones, dispositivos que también permitirían reducir los retrasos en la atención a los pacientes y mejoraría la continuidad asistencial.

La empresa norteamericana iHealth Lab Inc. sigue la línea de la mHealth y ha desarrollado dispositivos portables como pulsioxímetros, monitores de presión arterial y glucómetros compatibles con dispositivos móviles Apple a través de comunicación bluetooth.

Entonces, la implementación de un equipo portátil que permita visualizar las señales y variables biomédicas en dispositivos móviles Android como celulares o tablets es una excelente alternativa tecnológica frente a algunos equipos médicos de este tipo, ya que sería adecuada para monitorear los signos vitales de un paciente que se encuentra en casa, brindándole mayor comodidad. En este contexto, el objetivo es diseñar un sistema de oximetría de pulso y capnografía basado en un microcontrolador configurable llamado PSoC, que permita la medición de los niveles de $\mathrm{SpO}_{2}, \mathrm{EtCO}_{2}$, frecuencia cardiaca y frecuencia respiratoria en tiempo real, y la transmisión de las señales biomédicas usando tecnología inalámbrica WiFi, hacia dispositivos móviles, como smartphones o tablets Android.

El objetivo del diseño e implementación de este sistema no es obtener un equipo que compita con otros ya posicionados en el mercado, sino desarrollar una alternativa tecnológica flexible de monitoreo con la cual se pueda obtener un mayor provecho de los dispositivos móviles, además de explotar la tecnología de señal mixta del PSoC, y que sirva de plataforma base para el desarrollo de un monitor de signos vitales portátil, compatible con diferentes plataformas móviles y monitorización remota.

\section{Materiales y MÉTodos}

\subsection{Materiales}

El sistema de oximetría y capnografía está conformado por una unidad hardware de adquisición, procesamiento $\mathrm{y}$ transmisión de datos, y una interfaz de usuario que muestra en tiempo real las señales de los pacientes. 


\section{- Hardware}

La unidad hardware se diseñó y se implementó sobre una Tarjeta de circuito impreso (PCB: Printed Circuit Board) de doble cara, donde el elemento central de procesamiento es el circuito programable de señal mixta PSoC5 de Cypress semiconductor [5], en conjunto con componentes electrónicos de montaje superficial. En este caso, se emplearon diodos, transistores MOSFET, condensadores y un puente $\mathrm{H}$ integrado para la adecuación y alimentación del sensor Nellcor de $\mathrm{SpO}_{2}$ [6]. Para el capnógrafo se empleó el módulo $\mathrm{EtCO}_{2} \mathrm{C} 200$ fabricado por la empresa Goldwei [7]. La transmisión de los datos vía WiFi usa el módulo Wifly $\mathrm{RN}-\mathrm{XV}$ de comunicación inalámbrica [8]. La aplicación Android desarrollada se ejecuta en una Tablet Toshiba Excite 10.1" con Android 4.0.1.

En la Fig. 1 se muestra el diagrama general del sistema, el cual tiene 4 bloques funcionales: sensado, adquisición y procesamiento, comunicación y visualización.

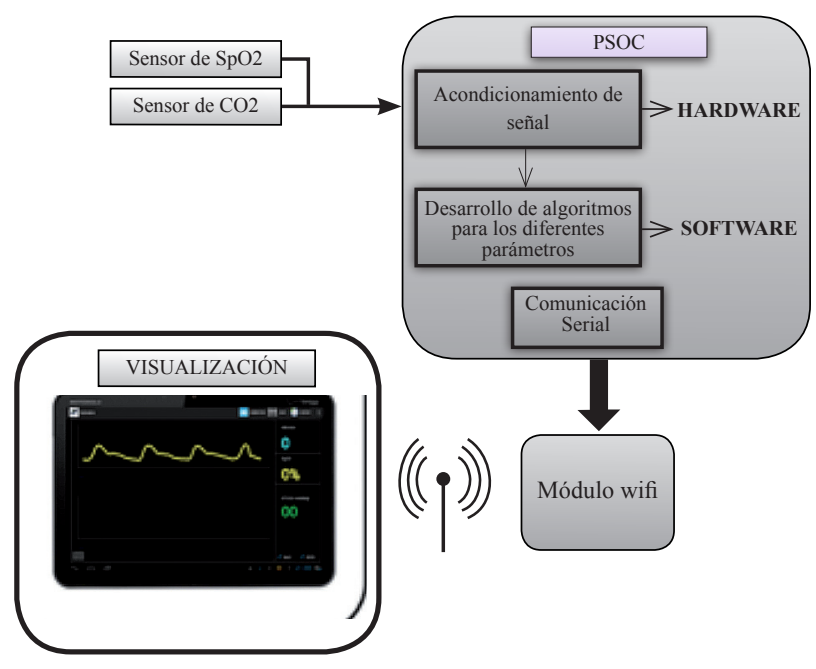

Fig. 1. Diagrama general del sistema.

\section{- $\quad$ Software}

El programa PSoC Creator 2.0 de Cypress semiconductor es utilizado para la compilación y programación en el PSoC5. El diseño y desarrollo de la interfaz de usuario se llevó a cabo con la herramienta Android developer tools basada en eclipse, y el Android SDK.

\subsection{Métodos}

- Oxímetro de pulso y principio de funcionamiento

La oximetría de pulso proporciona una medición del porcentaje de hemoglobina oxigenada (saturación de $\mathrm{O}_{2}$ en sangre) de forma continua, inmediata y no invasiva.
El principio de operación de la oximetría de pulso se basa en la ley de Beer-lambert (1) que establece que la absorbancia de la luz está directamente relacionada con las propiedades intrínsecas del analito, con su concentración y con la longitud de la trayectoria del haz de radiación al atravesar la muestra. Es importante resaltar que esta ley es válida inclusive si más de una sustancia absorbe luz en el medio [9].

La atenuación total de luz puede ser descrita por 4 componentes de absorbancias presentes en la sangre:

- Hemoglobina oxigenada (concentración $\mathrm{c}_{\mathrm{HbO}_{2}}$, coeficiente de absorbancia $\alpha_{\mathrm{HbO}_{2}}(\lambda)$ y longitud de trayectoria $\mathrm{d}_{\mathrm{HbO}_{2}}$ )

- Hemoglobina desoxigenada (concentración $\mathrm{c}_{\mathrm{Hb}}$, coeficiente de absorbancia $\alpha_{\mathrm{Hb}}(\lambda)$ y longitud de trayectoria $\left.\mathrm{d}_{\mathrm{Hb}}\right)$.

- Absorbancias específicas que no están en la sangre arterial (concentración $c_{x}$, coeficiente de absorbancia $\alpha_{\mathrm{x}}(\lambda)$ y longitud de trayectoria $\mathrm{d}_{\mathrm{x}}$ ).

- Otras fuentes de atenuación ópticas como factores geométricos, características de emisión y detección entre otras $\left(\mathrm{A}_{0}\right)$.

En este caso, la absorbancia total se puede ver como la sumatoria de las absorbancias parciales presentes en el medio (1).

$$
\begin{aligned}
& \mathrm{A}_{\mathrm{T}}=\alpha_{\mathrm{HbO}_{2}}(\lambda) \mathrm{c}_{\mathrm{HbO}_{2}} \mathrm{~d}_{\mathrm{HbO}_{2}} \\
& +\alpha_{\mathrm{Hb}}(\lambda) c_{\mathrm{Hb}} d_{\mathrm{Hb}}+\alpha_{\mathrm{x}}(\lambda) c_{\mathrm{x}} d_{\mathrm{x}}+\mathrm{A}_{0}
\end{aligned}
$$

Partiendo del hecho de que la luz roja visible es absorbida en mayor cantidad por la HbR (hemoglobina reducida o desoxigenada) que por la $\mathrm{HbO}_{2}$ (hemoglobina oxigenada), la cual absorbe en mayor cantidad la luz infrarroja, entonces la medida de la saturación de oxigeno está determinada por la cantidad de luz tanto roja como infrarroja que se puede proyectar a través del árbol arterial [10].

\section{- $\quad$ Diseño del Oxímetro}

Como se ha descrito previamente, el funcionamiento de la oximetría de pulso está basado en la medición de la absorción de luz roja e infrarroja, por lo que se usa un fotodiodo para recibir la luz, y dependiendo de la intensidad se genera una corriente en el fotodiodo, la cual se adecúa para su procesamiento y así determinar el nivel de $\mathrm{SpO}_{2}$. 


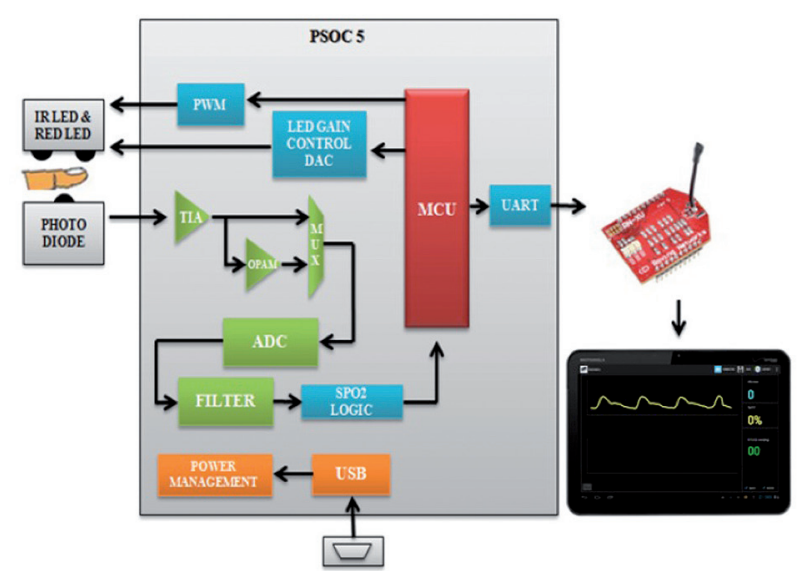

Fig. 2. Diagrama Funcional $\mathrm{SPO}_{2}$

La Fig. 2 muestra el esquema general del pulsioxímetro, que se encuentra embebido en el PSoC, tanto el bloque de adecuación de señal como el bloque de procesamiento; la etapa de alimentación de los LEDs es controlada por medio del PSoC. El diseño tiene una etapa de recepción de los pulsos de luz roja e infrarroja que pasan a través del dedo del paciente y son recibidos por un fotodiodo, el cual produce corriente dependiendo de la intensidad de los pulsos recibidos. Esta corriente es convertida en voltaje y amplificada, para luego ser digitalizada por el ADC, el cual es conmutado a la frecuencia de los pulsos de los LEDs, de forma que pueda medir por separado la intensidad de la luz roja e infrarroja. Las muestras digitalizadas por el ADC pasan a través de un filtro FIR pasa bajos, para atenuar el ruido introducido por lámparas de iluminación y la red de potencia $\mathrm{AC}$ de $60 \mathrm{~Hz}[2]$.

\section{A. Señal de excitación y control de intensidad}

La señal de excitación de los LEDs son pulsos generados por dos bloques PWM (Pulse Width Modulation) del PSoC, los cuales son programados para tener una señal PWM con periodo $t m=2 \mathrm{~ms}$ y un ciclo de trabajo del $25 \%$. Una señal PWM se desfasa $1 \mathrm{~ms}$. con respecto a la otra para permitir el encendido de un LED a la vez, y así obtener muestras de ambos LEDs por separado [12].

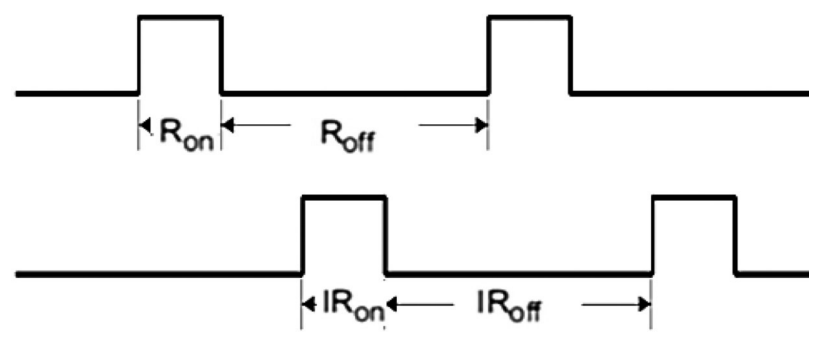

Fig. 3. Señal de Excitación de los LEDs.
También se implementó una fuente de corriente para realizar el control de intensidad de los LEDs a partir de MOSFETs, donde el voltaje de puerta se controla automáticamente dependiendo de la intensidad recibida, ya que esta puede variar de una persona a otra, debido a diferente densidad ósea, color de piel, etc.

\section{B. Amplificador de transimpedancia}

Este es un amplificador de realimentación negativa que se encarga de convertir la corriente producida por el fotodiodo en voltaje [13]. Consiste básicamente de una fuente de corriente (en este caso la producida por el fotodiodo) y un amplificador operacional. En este caso, una resistencia de $4,7 \mathrm{M} \Omega$ y un capacitor de $3 \mathrm{pF}$ son conectados en paralelo entre la entrada y la salida del amplificador embebido en el PSoC.

\section{Procesamiento de la señal}

La señal proveniente desde cada LED es filtrada usando un FIR paso bajos con $\mathrm{Fc}=30 \mathrm{~Hz}$, y posteriormente esta señal es filtrada usando un IIR paso bajos descrito en (2) con $\mathrm{Fc}=0,5 \mathrm{~Hz}$ para obtener la componente DC de la señal.

$$
y(n)=\frac{x(n)+x(n-1)-y(n-1) \times(1-k)}{1+k}
$$

Donde

$$
\begin{array}{r}
k=\frac{1}{2 \pi f c}\left(\frac{2}{t m}\right) \\
\text { Siendo } f_{c}=0.5 H z \text { y } \mathrm{tm}=2 \mathrm{~ms}, \\
k=318.309
\end{array}
$$

La oximetría de pulso hace referencia a la medición del porcentaje de oxígeno en cada pulsación de la sangre arterial. Para realizar esta medición es necesario operar las componentes AC y DC de la señal pulsátil producida por cada LED.

Por lo tanto, el cálculo del $\mathrm{SpO}_{2}$ consiste en utilizar las señales temporales de luz roja e infrarroja para calcular un valor llamado "R" descrito en (4), que corresponde a la relación normalizada de las intensidades de luz roja e infrarroja recibidas por el fotodiodo.

$$
R=\frac{\frac{A C_{R}}{D C_{R}}}{\frac{A C_{I R}}{D C_{I R}}}
$$

Donde $A C_{R}-D C_{R}$ y $A C_{I R}-D C_{I R}$ corresponden a la componente $\mathrm{AC}$ y $\mathrm{DC}$ de la señal roja e infrarroja respectivamente.

Para calcular el valor RMS de la señal AC se emplea (5). 


$$
V_{R M S}=\sqrt{\left(\frac{1}{T}\right) \int_{0}^{\mathrm{T}} V^{2}(t) d t}
$$

Donde la integral se calcula empleando la regla del trapecio, de acuerdo a (6).

$$
\int_{a}^{b} f(x) d x \cong \frac{b-a}{2}[f(a)+f(b)]
$$

Para garantizar que la integral se lleve a cabo solo a una onda pulsátil, el intervalo [a,b] es modificado de acuerdo al pulso cardiaco, el cual es determinado en los puntos máximos de la onda pulsátil. Para calcular adecuadamente la $\mathrm{SpO}_{2}$, es necesario encontrar la función que relaciona la saturación de oxigeno con el valor de $\mathrm{R}$ calculado, por medio del simulador de paciente.

La frecuencia cardiaca se determina calculando la derivada de la señal pulsátil generada por la incidencia del haz de luz infrarroja, y multiplicando esta por una constante, lo cual permite comparar la señal derivada con un valor establecido. Si la derivada supera el valor umbral establecido, un temporizador es activado para medir el tiempo transcurrido entre latidos. Este tiempo es del orden de minutos para medir el número de latidos por minuto [2].

\section{- Capnógrafo y principio de funcionamiento}

Un capnógrafo es un equipo electrónico portátil utilizado para la medición y registro continuo del dióxido de carbono espirado; la capnografía es la monitorización no invasiva del $\mathrm{CO}_{2}$, donde la técnica de absorción de luz infrarroja es la más usada y se basa en la absorción de luz infrarroja por las moléculas de $\mathrm{CO}_{2}$ en una muestra de gas espirado. La capnografía aporta datos sobre la producción de $\mathrm{CO}_{2}$, la perfusión pulmonar, la ventilación alveolar y el patrón ventilatorio.

La fracción de $\mathrm{CO}_{2}$ medida al final de la espiración de un volumen tidal se aproxima mucho a la concentración alveolar de $\mathrm{CO}_{2}$, porque esta se mide cuando el paciente espira prácticamente gas alveolar puro (gas alveolar que está en equilibrio con la sangre capilar pulmonar). Así, el $\mathrm{EtCO}_{2}$ (end-tidal $\mathrm{CO}_{2}$ ) se considera una medida indirecta de la presión arterial de $\mathrm{CO}_{2}$. Bajo circunstancias normales la diferencia de $\mathrm{EtCO}_{2}$ y presión arterial de $\mathrm{CO}_{2}$ es de (2-7) $\mathrm{mmHg}$, y el rango normal de $\mathrm{EtCO}_{2}$ para una persona sana está entre (33-39) $\mathrm{mmHg}$ [14].

\section{- Descripción del Sensor}

Para este diseño se decidió utilizar el Módulo OEM $E t C O$, Model C200 fabricado por la empresa Goldwei. El sensor de este módulo es NDIR (Non Dispersive InfraRed), posee una exactitud de $\pm 2 \mathrm{mmHg}$ y mide la frecuencia respiratoria en un rango de 2-150 BPM (Breaths per Minute). En la Fig. 4 se puede observar el módulo utilizado.

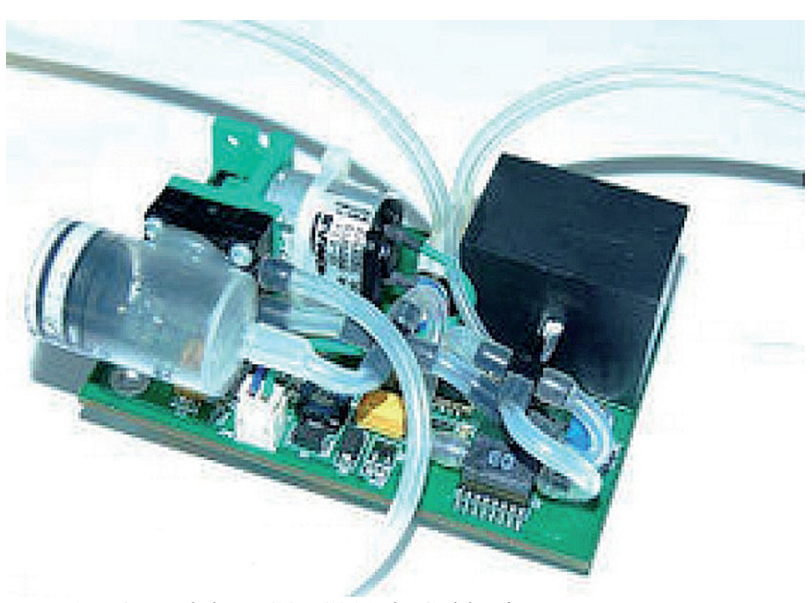

Fig. 4. Módulo $\mathrm{EtCO}_{2} \mathrm{C} 200$ de Goldwei.

\section{- Implementación del Capnógrafo}

El capnógrafo implementado es tipo sidestream, en el cual una muestra de $\mathrm{CO}_{2}$ es aspirada para ser analizada, en este caso por un sensor infrarrojo no dispersivo.

Este módulo toma continuamente el gas exhalado del paciente a través de una cánula nasal que canaliza el aire expirado desde el interior de las fosas nasales. El gas es extraído por medio de una bomba extractora de tal forma que el gas que pasa por el sensor tenga un flujo constante [15].

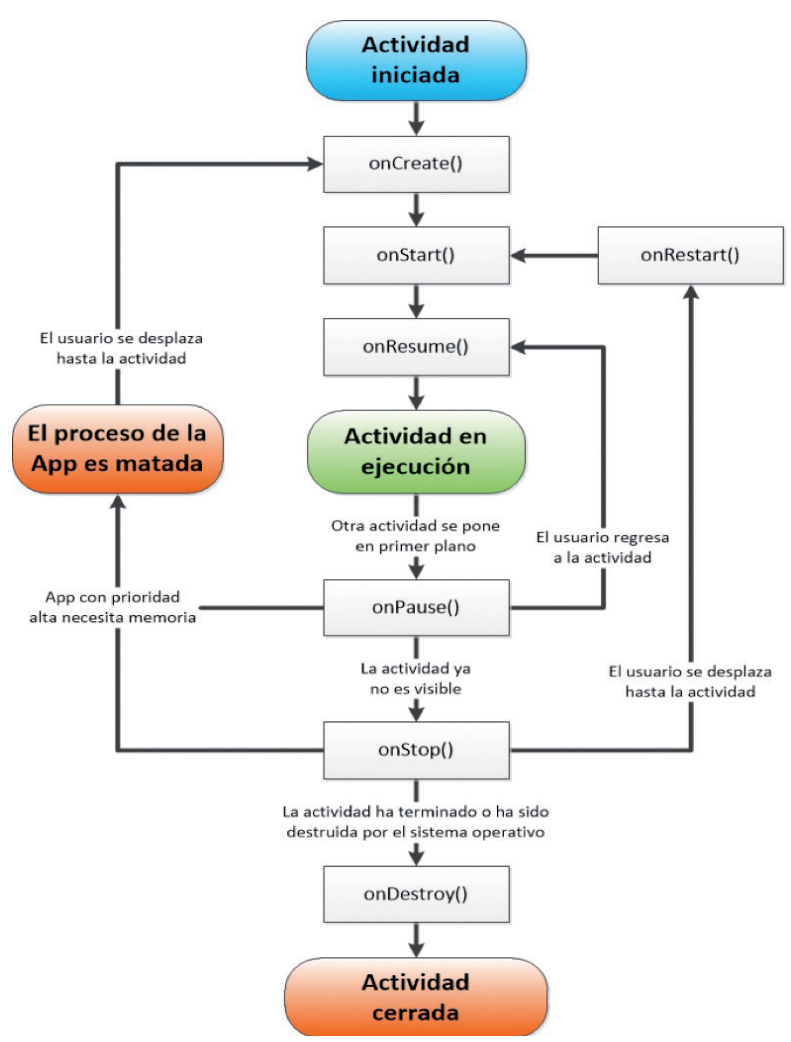

Fig. 5. Ciclo de vida de una Actividad. 
Los datos del nivel de $\mathrm{EtCO}_{2}$ y la frecuencia respiratoria son enviados al PSoC a través de interfaz serial UART y desde el PSoC se envían los datos al módulo de comunicación wifi para transmitirlo al dispositivo Android.

\section{- Interfaz de Usuario}

El diseño de la interfaz gráfica de usuario se realizó utilizando la plataforma de desarrollo de Android, basado en el lenguaje de programación Java por medio de la herramienta Eclipse para su compilación y depuración.

La ejecución de una aplicación en Android se comporta como una máquina de estados, llamada ciclo de vida, y teniendo en cuenta ese comportamiento se programa la aplicación. Cuando se habla de ciclos de vida en una aplicación en Android, específicamente se refiere al ciclo de vida de una app propiamente, y al ciclo de vida de cada una de las actividades (Activity: clase principal en ejecución) que la componen. Una aplicación está compuesta por una o más actividades (que en este caso interactúan entre sí) y realiza un "camino" desde el momento que se lanza hasta que se cierra por completo. Este camino se inicia luego de lanzar la aplicación (abrirla desde el dispositivo), con lo cual va automáticamente a la actividad que se marca inicialmente como launcher (en el archivo Manifest se puede encontrar en la lista de activities, marcada con un intent-filter). De ahí en adelante, el ciclo de vida de la aplicación pasa a mano de las actividades que la componen, cada una de las cuales cuenta con un ciclo de vida propio, tal como se puede observar en la Fig. 5 [20].

La aplicación tiene dos clases principales que se encargan de recibir los datos y graficarlos; una se encarga de la comunicación TCP/IP entre la Tablet y la unidad hardware de medición. La otra clase, tipo "Activity", se encarga de la interfaz de usuario y la visualización en tiempo real de la onda pletismográfica y la onda de capnografía. Tiene además otras tres clases tipo "Activity", encargadas de la ventana de inicio de sesión, la ventana de registro de usuario y la ventana de historial, donde se muestran valores almacenados en el tiempo de $\mathrm{SpO}_{2}, \mathrm{EtCO}_{2}$, HR y frecuencia respiratoria.

Para intercambiar los datos con la unidad hardware de medición, la aplicación realiza la apertura de sockets para dejar pasar por el puerto de comunicación los datos que son enviados desde el módulo wifi del hardware de medición a través del protocolo TCP/IP. Estos datos son almacenados en Buffers para luego ser utilizados por el método de graficación, implementado mediante la librería Achartengine, la cual proporciona gran flexibilidad en el desarrollo de diagramas X-Y y diagramas de barra, tanto dinámico como estático. En la Fig. 6 se muestra el diagrama de flujo del funcionamiento de la aplicación.

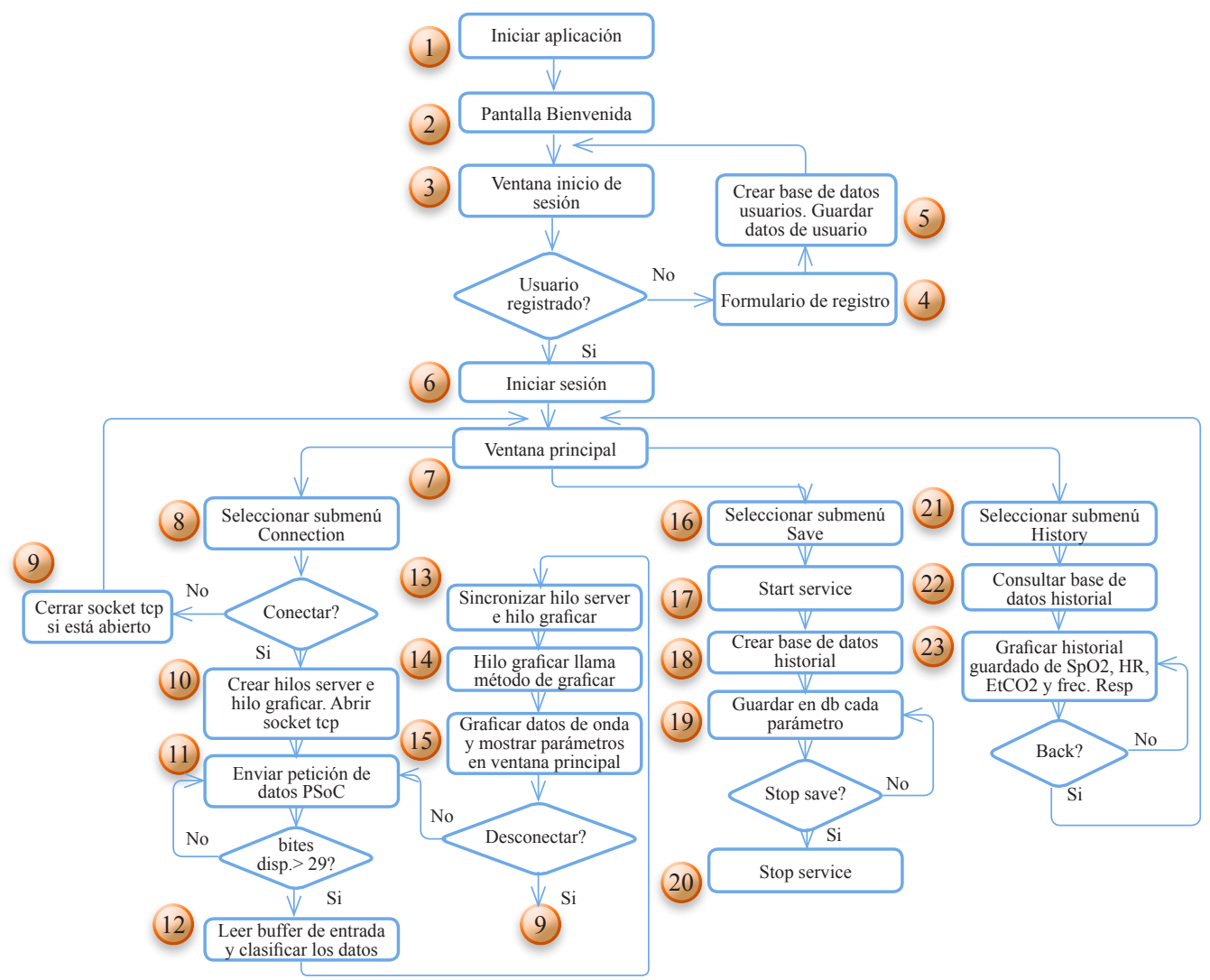

Fig. 6. Diagrama de flujo de la aplicación para la interfaz de usuario 
La apariencia de la aplicación, es decir, la forma, color, campos y botones son originados a partir de archivos xml, que interactúan con las "Activity" definidas en la clase principal para modificar dinámicamente su aspecto $\mathrm{y}$ valores de los campos.

\section{RESUltados}

Luego del diseño e implementación de las unidades de hardware y software mencionados anteriormente, se obtuvo un prototipo de pulsioximetría y capnografía, capaz de realizar la medición de $\mathrm{SpO}_{2}$, frecuencia cardiaca $\mathrm{HR}$ (Heart Rate), $\mathrm{EtCO}_{2}$ y frecuencia respiratoria en tiempo real, permitiendo visualizar todas las variables y señales en una interfaz gráfica realizada para dispositivos Android.

\section{- $\quad$ Características de la interfaz de usuario}

La aplicación de interfaz de usuario desarrollada para dispositivos Android, abre en primera instancia una pantalla de ingreso de usuario y contraseña. Si el usuario no está registrado podrá hacerlo haciendo clic en el botón "Registrar", el cual lo llevará a un formulario de registro donde ingresará sus datos para ser almacenados. Después de haber ingresado como usuario usando la contraseña se despliega la pantalla principal de la aplicación. La mayor parte del área de la pantalla es usada para graficar tanto la onda pletismográfica de pulsioximetría, como la onda de respiración del capnógrafo. En la parte derecha se muestran los siguientes valores en orden descendente: frecuencia cardiaca (HR), saturación de oxigeno $\left(\mathrm{SpO}_{2}\right)$, $\mathrm{EtCO}_{2}$ y frecuencia respiratoria.

En la parte superior se encuentra el botón "Conexión", para comunicar la aplicación con la unidad hardware, el botón "Guardar" para almacenar los valores de las mediciones recibidas cada cierto tiempo en una base de datos, y el botón "Historial" para mostrar en gráficos temporales, los valores de las mediciones guardadas de cada paciente. En la Fig. 7 y la Fig. 8 se muestran las ventanas de la interfaz de usuario.

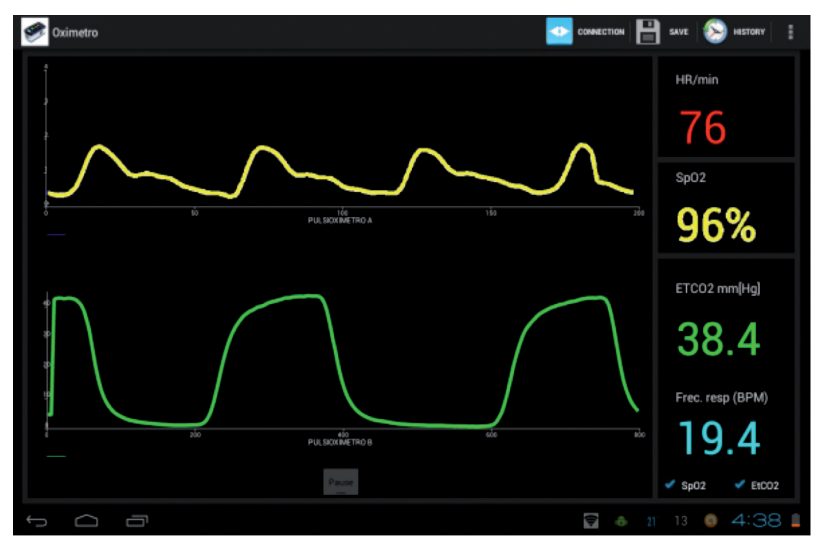

Fig. 7. Ventana principal interfaz de usuario

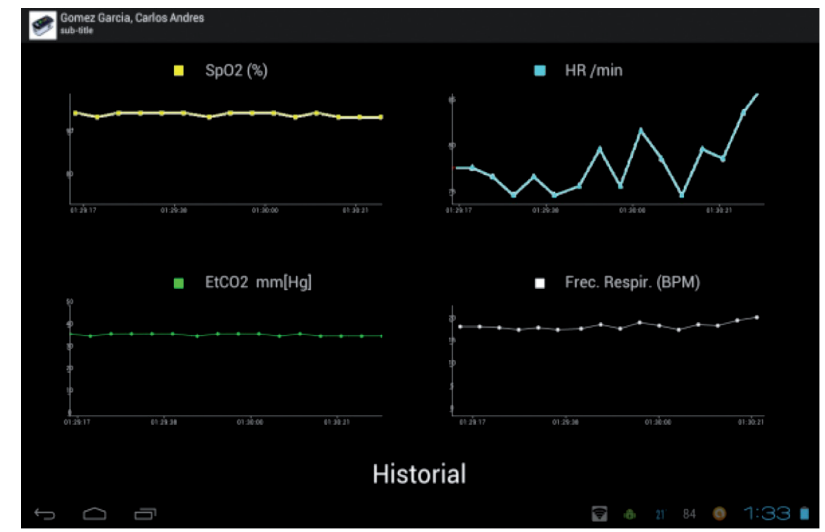

Fig. 8. Ventana de historial de señales de un paciente

- $\quad$ Pruebas utilizando el simulador de pacientes

El simulador de $\mathrm{SpO}_{2}$ FLUKE Index $2 X L$ fue utilizado para la calibración y posterior verificación del pulsioxímetro. Por medio de este simulador se halló la ecuación (7) que relaciona el valor $\mathrm{R}$ con la $\mathrm{SpO}_{2}$, mediante la variación del valor de $\mathrm{SpO}_{2}$ desde $75 \%$ hasta $100 \%$, y hallando el respectivo valor de $\mathrm{R}$.

$$
\mathrm{SpO}_{2}=103,05-10,64 R
$$

Una vez calibrada la unidad hardware de pulsioximetría, se procedió a realizar las pruebas de verificación de la medición de $\mathrm{SpO}_{2}$ y $\mathrm{HR}$ con el simulador. En la Tabla 1 se muestran los resultados de la medición de $\mathrm{SpO}_{2}$ a frecuencia constante de 70 BPM.

Tabla 1. Resultados de validación de $\mathrm{SpO}_{2}$ con el simulador

\begin{tabular}{ccc}
\hline $\mathrm{SpO}_{2}$ simulado (\%) & $\mathrm{SpO}_{2}$ medido (\%) & Error relativo (\%) \\
\hline 75 & 76 & 1,33 \\
80 & 81 & 1,25 \\
85 & 84 & 1,18 \\
90 & 89 & 1,11 \\
95 & 94 & 1,05 \\
100 & 100 & 0,00 \\
\hline
\end{tabular}

En la Tabla 2 se muestran los valores de las mediciones obtenidas para el número de pulsos por minuto para un nivel de $\mathrm{SpO}_{2}$ constante de 95\%.

Tabla 2. Resultados de validación de frecuencia cardiaca con el simulador

\begin{tabular}{ccc}
\hline Pulso simulado (BPM) & Pulso medido (BPM) & Error relativo (\%) \\
\hline 55 & 56 & 1,81 \\
65 & 64 & 1,54 \\
75 & 74 & 1,33 \\
85 & 86 & 1,18 \\
95 & 94 & 1,05 \\
105 & 104 & 0,95 \\
115 & 116 & 0,86 \\
125 & 125 & 0,0 \\
135 & 137 & 1,48 \\
145 & 147 & 1,37 \\
\hline
\end{tabular}


Como el sensor de $\mathrm{EtCO}_{2}$ esta calibrado de fabrica, no se realizo su validación con equipo especializado, sin embargo se realizaron mediciones de nivel $\mathrm{EtCO}_{2}$ y frecuencia respiratoria a 5 sujetos sanos en reposo, obteniendo un rango normal de (36-39) $\mathrm{mmHg}$ de $\mathrm{EtCO}_{2} \mathrm{y}$ frecuencia respiratoria entre (13-22) BPM. Las mediciones respectivas de cada sujeto se muestran en la Tabla 3.

Tabla 3. Resultados de medición de $\mathrm{EtCO}_{2}$ a 5 personas

\begin{tabular}{lccccc}
\hline & $\begin{array}{c}\text { Edad } \\
\text { (años) }\end{array}$ & Peso (Kg) & $\begin{array}{c}\text { Estatura } \\
(\mathrm{m})\end{array}$ & $\begin{array}{c}\mathrm{EtCO}_{2} \\
(\mathrm{mmHg})\end{array}$ & $\mathrm{BPM}$ \\
\hline Sujeto 1 & 24 & 82 & 1.75 & 38.5 & 19 \\
Sujeto 2 & 32 & 75 & 1.63 & 37 & 17 \\
Sujeto 3 & 60 & 85 & 1.60 & 36.5 & 13 \\
Sujeto 4 & 55 & 65 & 1.60 & 39 & 15 \\
Sujeto 5 & 10 & 45 & 1.45 & 38 & 22 \\
\hline
\end{tabular}

\section{DisCUSIÓN}

El sistema electrónico correspondiente al pulsioxímetro presenta características comparables con otros desarrollos respecto a la frecuencia de muestreo y técnicas de filtrado y control $[10,11]$. La precisión en la medición tanto de $\mathrm{SpO}_{2}$ como de la frecuencia cardiaca es de \pm 2 dígitos, similar a equipos comerciales [6].

El desarrollo de este sistema sienta las bases para el diseño de futuros equipos y aplicaciones mHealth, que permitan el monitoreo remoto, el tele-diagnóstico en tiempo real y la conexión de profesionales de la salud con sus pacientes. Existen algunos dispositivos y aplicaciones de este tipo en el mercado, en su gran mayoría para móviles Apple, como los comercializados por la empresa iHealth, pero muy pocos compatibles con móviles Android, los cuales conforman el $80 \%$ de la cuota de mercado en smartphones.

Para lograr un equipo más competitivo desde el punto de vista funcional, se deben realizar algunas mejoras al sistema actual, como alimentar la tarjeta electrónica con baterías LiPo de $3.7 \mathrm{~V}$, y modificar la tarjeta para permitir recargarlas; incluir alarmas visuales y sonoras a la aplicación Android con el propósito de indicar anomalías en la medición; acondicionar el firmware del PSoC y la aplicación Android, para permitir la monitorización remota a través de internet.

En el futuro se pretende adicionar otras señales biomédicas al sistema, como las señales ECG y la presión arterial, ya que una de las motivaciones principales para el desarrollo del sistema descrito en este artículo es lograr un prototipo completo de tele-monitoreo, el cual puede contribuir al mejoramiento de la atención medica en los centros de salud de nivel 1 y 2, logrando la disminución de tiempos de atención médica, diagnóstico y tratamiento más oportuno, reduciendo el problema de sobre costos por transporte, escasez de especialistas y congestión en centros hospitalarios, brindando mayor cobertura y beneficiando a los pacientes que realmente necesitan ser atendidos. Según estudios de Cisco, cerca del $85 \%$ de las consultas médicas no requieren de una interacción física entre médicos y pacientes, por lo que la Telemedicina, al masificarse, podría generar numerosos beneficios [16].

\section{Conclusión}

En este trabajo se diseñó e implementó un sistema de medición de señales de pulsioximetría y capnografía portátil, que permite la monitorización de pacientes usando dispositivos móviles Android, así como la supervisión de los signos vitales de cada paciente en el tiempo. En relación a la precisión de las mediciones de las señales del pulsioxímetro, se calculó que la medición de la frecuencia cardiaca presenta un error de $\pm 1,81 \%$ y la medición de $\mathrm{SpO}_{2}$ presenta un error de $\pm 1,33 \%$.

Este sistema está diseñado para ser compatible con sensores tipo Nellcor que tengan conector DB9, lo cual permite un fácil reemplazo del mismo, debido a que es un sensor comercial y de fácil adquisición.

El chip de procesamiento de señal mixta (PSoC) utilizado en el sistema desarrollado tiene un gran valor agregado, al permitirle al diseñador hacer modificaciones o adiciones al sistema sin realizar mayores cambios al hardware, reduciendo así el tiempo de diseño. El sistema cuenta con un conector JTAG para la programación y depuración, brindando flexibilidad para implementar futuros desarrollos.

\section{REFERENCIAS}

[1]. Sánchez M. D. Procesado y transmisión de señales biomédicas para el diagnóstico de trastornos y enfermedades del sueño. Tesis doctoral. Escuela superior de ingeniería. Cadiz-España, 2008.

[2]. Gómez C.A, Martínez S.J. Diseño e implementación de un microsistema para medir gases en la sangre. Tesis de Grado. Ingenieros electrónicos, Universidad del Valle, 2012.

[3]. Ansermino J.M. Universal Access to Essential Vital Signs Monitoring. Anesthesia \& Analgesia Journal, 117, Octubre 2013.

[4]. Patel B.K., Chapman C. G., Luo N., Woodruff J.N., Arora V.M. Impact of Mobile Tablet Computers on Internal Medicine Resident Efficiency. Archives of Internal Medicine, 172, Marzo 2012.

[5]. Cypress Semiconductor. CY8C5568AXI-060-Programmable System-on-Chip (PSoC) DC to $67 \mathrm{MHz}$ operation Datasheet. Consultado el 1 de agosto de 2013 en: http://ecologylab.net/ courses/sensoryInterfaces/resources/CY8C55DataSheet.pdf.

[6]. Nellcor. Nellcor ${ }^{\mathrm{TM}}$ with OxiMax ${ }^{\mathrm{TM}}$ Technology. Product Guide. Consultado el 1 de noviembre de 2012 en: http://www.covidien. com/imageServer.aspx/doc229982.pdf?contentID=30898\&conten ttype $=$ application/pdf.

[7]. Goldwei. User's Guide for EtCO2 Module Model C002, C002a. Consultado el 20 de enero de 2013 en: http://www.goldwei. com/docs/ETCO2_MODULE_MOC002_USER_GUIDE_ revised-3-2-2010.pdf. 
[8]. Roving Networks, Inc. User Manual and Command Reference WiFly GSX/EZX. Consultado el 10 de febrero de 2013 en: http:// dlnmh9ip6v2uc.cloudfront.net/datasheets/Wireless/WiFi/WiFlyRN-UM.pdf.

[9]. Ventosinos S.A. Sistema óptico autónomo para la medida de parámetros. Tesis de Grado. Universidad Politécnica de Cataluña, 2010.

[10]. Matviyenko S. Cypress Semiconductor. Pulse Oximeter Application Note AN2313. Consultado el 1 de agosto de 2013 en: http://www.psocdeveloper.com/uploads/tx_piapappnote/ an2313_01.pdf.

[11]. Chan V., Underwood S. Application Report SLAA274A: A Single-Chip Pulsoximeter Design Using the MSP430. Consultado el 1 de agosto de 2013 en: http://www.ti.com/lit/an/slaa274b/ slaa274b.pdf.

[12]. Rivera I.Z., Herrera J.C., Ochoa V.J. Oxímetro de Pulso basado en una palm. VI Ciclo y 2do Congreso Nacional Tendencias Tecnológicas en Computación. México, D.F., noviembre 2006.

[13]. Baltazar López R.F. Fotodetector basado en un dispositivo p-i-n y un Amplificador de Transimpedancia. Segundo Congreso Nacional de Electrónica. Puebla, Pue. México, septiembre 2002.

[14]. Becerra Velásquez R, Tantaleán J. Comparación de $\mathrm{CO}_{2}$ al final de la espiración y presión arterial de $\mathrm{CO}_{2}$ en pacientes pediátricos con ventilación mecánica. Revista Peruana de Pediatría, septiembre-diciembre 2005.

[15]. Córdova J.A, Ortiz M.E, Velázquez A. Centro Nacional de Excelencia Tecnológica en Salud CENETEC, México. Guía Tecnológica No 37: Capnógrafo. Consultado el 25 de noviembre de 2012 en:http://www.cenetec.salud.gob.mx/descargas/equipo guias/guias_tec/37gt_Capnografia.pdf.

[16]. CISCO. La solución Cisco HealthPresence: los límites de la atención sanitaria. Consultado el 1 de agosto de 2013 en: http://www.cisco.com/web/ES/solutions/industria/cisco_ healthpresence_solution.html

[17]. Di G., Tang X., Liu W. A Reflectance Pulse Oximeter Design Using the MSP430OF149. IEEE/ICME International conference on Complex Medical Engineering, Beijing, China, 2007.

[18]. Guyton A.C., Hall J.E. Tratado de Fisiología Médica. McGrawHill, 2001. Capítulos 39, 40.

[19]. Rüdiger M., Töpfer K., Hammer H., Schmalisch G., Wauer R. Estudio de vigilancia de los gases en sangre transcutánea entre las unidades de cuidados intensivos neonatales. BMC Pediatric, 5(30), 2005

[20]. Gargenta M. Learning Android. Building Applications for the Android Market. O'Reilly Media, 2011. Capítulo 4, 5, 7, 8, 9, 10. 